\title{
Pulmonary Metastasectomy in Colorectal Cancer (PulMiCC): a multi-centre randomized clinical trial
}

\section{Tom Treasure ( $\nabla$ tom.treasure@gmail.com )}

University College London https://orcid.org/0000-0001-9358-7610

\section{Vern Farewell \\ MRC Cambridge}

\section{Fergus Macbeth}

Cardiff University

Kathryn Monson

University of Sussex

Norman R Williams

University College London

\section{Chris-Brew Graves}

University College London

\section{Belinda Lees}

University of Oxford

\section{Olivia Grigg}

University of Cambridge

\section{Lesley Fallowfield}

SHORE-C University of Sussex

\section{Research}

Keywords:

Posted Date: July 19th, 2019

DOl: https://doi.org/10.21203/rs.2.11719/v1

License: (c) (i) This work is licensed under a Creative Commons Attribution 4.0 International License. Read Full License

Version of Record: A version of this preprint was published on December 12th, 2019. See the published version at https://doi.org/10.1186/s13063-019-3837-y. 


\section{Abstract}

Background: Lung metastasectomy in the treatment of advanced colorectal cancer has been adopted and established without control data. Our aim was to test its effectiveness in a randomised trial. Methods: Multidisciplinary teams in 13 hospitals recruited participants with potentially resectable lung metastases to a multicentre 2-arm randomised trial comparing active monitoring with or without metastasectomy. Other treatments were as decided by the local team. Randomization was centralised with stratification by site and minimisation for age, sex, primary cancer stage, interval since primary resection, prior liver involvement, the number of metastases, and carcinoembryonic antigen. The assigned arm was not disclosed to the trial management group until completion of analysis. Analysis was on intention to treat with a margin for non-inferiority of 10\%. Findings: Between December 2010 and December 2016, 65 participants were randomised. Characteristics were well-matched in the two arms and similar to those in reported studies: age 35 to 86 (IQR 60 to 74); primary resection IQR 16 to 35 months previously; stage at resection T1, 2 or 3 in 3, 8 and 46; N1 or N2 in 31 and 26; unknown in 8. Lung metastases 1 to 5 (median 2); 16/65 had previous liver metastases; carcinoembryonic antigen normal in $55 / 65$. There were no other interventions in the first 6 months, no cross overs from control to treatment, and no treatment related deaths or major adverse events. Hazard ratio for death within 5 years, comparing metastasectomy with control, was $0.82(95 \% \mathrm{Cl} 0.43,1.56)$. Interpretation: The small number $(\mathrm{N}=65)$ precludes a conclusive answer to the research question but the similar survival in metastasectomy and control arms challenges beliefs that there is a 35\% survival difference that can be attributed to lung metastasectomy. Funding: Cancer Research UK funding Grant No. C7678/A11393 Name of the registry: Clintrial.gov Registration number: NCT01106261 Date 19th April 2010 https://clinicaltrials.gov/ct2/show/NCT01106261

\section{Background}

Standards of care in colorectal cancer include detection by active surveillance of asymptomatic metastases followed by surgical resection in selected patients. Lung metastasectomy is now regarded as 'a pillar of modern thoracic surgery' ${ }^{1}$ and is a substantial component of the work of thoracic surgical units internationally. This activity has been reported increasingly in clinical case series from the 1960s. ${ }^{2}$ The publication of the International Registry of Lung Metastases in 1997 established lung metastasectomy in clinical practice. ${ }^{3}$ The report contains data on patients who had a lung metastasectomy performed by the contributing surgeons but, as is typical in procedure-based clinical reporting, there were no comparable data on those who did not have metastases removed. One small comparative study was published in $1980 .{ }^{4}$ It reported that the survival of 12 patients who were potential candidates for metastasectomy but did not have it was not dissimilar to 70 comparable patients who had lung metastasectomy. The number of metastasectomy operations continued to increase during the period 2000 to $2011^{5,6}$ without any randomised trials, a time when there were many controlled trials of systemic therapies. ${ }^{7}$ In 2013 a meta-analysis of the 25 largest single arm follow-up studies from 2000 to 2011 , reported an overall five-year survival rate of $41 \%$ for patients having lung metastasectomy for 
colorectal cancer, at an average interval of about two years after primary resection. No controlled studies were found and the authors concluded 'the benefit attributable to surgery is neither immediate nor irrefutable'. ${ }^{8}$

There is some indirect evidence from controlled trials that metastasectomy may not lengthen survival. There have been two meta-analyses of randomised trials comparing more with less intensive surveillance in patients treated for early colorectal cancer. Surveillance successfully advances detection: metastases were diagnosed up to two years earlier. There were more surgical interventions but there was no overall survival benefit. 9,10 There was also uncertainty expressed by the authors of a meta-analysis of colorectal cancer survival gains who noted 'that while indeed more metastasectomies are being performed, they have been made possible by better therapies and that this benefit should be ascribed to the therapies' thus raising the possibility of reverse causation. ${ }^{7}$ That is to say that longer survival provides opportunities for more treatments rather than additional treatments necessarily being the cause of longer survival.

Early expressions of doubt about the clinical effectiveness of lung metastasectomy pointed to the lack of control data ${ }^{4.11}$ but the weight of current opinion is that the observational evidence is sufficient. ${ }^{1}$ However, the proposed criteria set out for trusting clinical observation, without the need for a controls, are not met. ${ }^{12}$ The effect of the intervention has to be mechanistically plausible and a close temporal association between the intervention and the desired outcome is required. The variable course of cancer, and the deliberate selection of patients with very few metastases and a slower course, makes picking the signal from the noise impossible. ${ }^{12}$ The missing evidence is control data on the survival of patients with features making them eligible but who did not have metastasectomy. The need for this evidence led to the Pulmonary Metastasectomy in Colorectal Cancer (PulMiCC) randomised controlled trial which we report here.

\section{Methods}

Study design: PulMiCC was a two-stage randomized Phase III, parallel arm, multicentre trial.

The setting was hospital based multidisciplinary teams (MDTs) managing patients with advanced colorectal cancer. The Principal Investigator ( $\mathrm{Pl})$ at each trial site was a medical member of the team, either a surgeon or an oncologist. The study was set up in 24 hospitals treating advanced colorectal cancer: 21 were in Britain, with one each in Serbia, Italy and China. 
The trial was co-ordinated from October 2009 to March 2014 by the Clinical Trials and Evaluation Unit, Royal Brompton and Harefield NHS Foundation Trust, London. PulMiCC administration and trial management then moved to the Surgical and Interventional Trials Unit (SITU), University College London. Both units are subsequently referred to as 'the Trials Unit'. The co-ordination of patient reported outcomes (PROs) throughout was at Sussex Health Outcomes Research \& Education in Cancer (SHORE-C), University of Sussex.

Ethics Approval and Consent to Participate

Central ethical approval was confirmed from the National Research Ethics Committee London Hampstead (ref approval no. 10/H0720/5) and did not begin recruiting at other centres in the trial until local ethical approval was obtained. Written informed consent was obtained at enrolment (Stage 1) and separately at randomisation (Stage 2 ).

The trial protocol can be accessed on line.

https://www.ucl.ac.uk/clinical-operational-research-unit/

A description of the trial can be accessed on line.

https://clinicaltrials.gov/ct2/show/study/NCT01106261?show_desc=Y\#desc

\section{Patient participants}

Eligible for inclusion were adults who had undergone resection of primary colorectal cancer with a prospect of cure, but who now had pulmonary metastasis, confirmed on routine review by the multidisciplinary team (MDT). Previously treated liver metastases were allowed but there had to be no clinical indication of active colorectal cancer, by investigations including imaging by computerised tomography (CT) and positron emission tomography (PET). Exclusion criteria were previous malignancy, concurrent illness, or unavailability for follow-up that was likely to interfere with treatment per protocol or the measurement of endpoints, or if mental incapacity precluded fully informed consent.

Patient participants were recruited from the MDT meetings and registered for evaluation in Stage 1 after written informed consent. Those subsequently eligible for randomisation and for whom the MDT was in 
equipoise about the benefit of metastasectomy, were offered random assignment after written consent (Stage 2) to have metastasectomy or not with both arms receiving continued active monitoring.

Trial process: A designated clinical team member informed potentially eligible patients of the MDT findings and explained the trial, emphasising the uncertainty of the evidence for the management of pulmonary metastases. Those interested in participating were given a patient information leaflet and an explanatory digital video disc (DVD) to take home. A healthcare professional training DVD was also available for clinicians to aid their discussions with patients. The trial was administered by clinical trials staff based in the local hospital site under the direction of the PI. Medical MDT members provided information and dates of events and measurements appropriate to their specialization. These were collated locally and returned to the Trials Unit on clinical report forms [CRFs]. Once a patient consented to join Stage 1, registration was carried out by the Trials Unit.

Following evaluation and any systemic treatments considered appropriate, eligible patients were approached by the oncologist or other designated member of the clinical team and asked if they were willing to consider the second (randomised) stage of the trial. All patients eligible for Stage 2 of the trial, whether or not they had chosen to proceed to randomisation, were invited to complete a questionnaire exploring reasons for accepting or declining trials; this was completed at home and returned by post to SHORE-C. ${ }^{13}$

Patients who confirmed their willingness to be randomised were asked to sign a second consent form and complete a set of baseline questionnaires: Functional Assessment of Cancer Therapy - General and Anaemia sub-scale (FACT-G-An) ${ }^{14}$ plus selected items from the six item Lung Cancer Brief Symptom Index ${ }^{15}$ and the six item short form of the Spielberger State/Trait Anxiety Inventory (STAl). ${ }^{16}$ The EQ-5D$3 \mathrm{~L}$ (EuroQoL 5 Dimension 3 Level) questionnaire was administered for health economic evaluation. ${ }^{17}$ Details are provided as supplementary materials.

\section{Randomisation and masking}

Random assignment was to active monitoring (Control) or the same plus metastasectomy (Metastasectomy). 
Randomisation was stratified by local site. Patients were allocated equally between the treatment arms. Sequence generation was at www.sealedenvelope.co.uk using a minimisation program incorporating the participant's age, sex, $\mathrm{T}$ (umour) stage, $\mathrm{N}$ (odal) stage, prior liver metastasectomy, time since resection of the colorectal primary cancer, number of metastases, and CEA level. A random element was included such that each patient retained a non-zero probability of being randomised to each of the treatment arms. The trials staff transmitted the request and received the assignment electronically. Because the assignment was performed remotely, the randomisation was completely independent of the investigators and the Trials Unit.

Allocation concealment from the participants or the site staff was not possible. The Trial Management Group (TMG) remained completely blind to assignment until after the primary analysis was done and the trial statistician and the TMG agreed the release of the full database for further analysis.

\section{Procedures}

Control participants were to be managed without metastasectomy, radiotherapy or image guided thermal ablation (IGTA). If these treatments were used subsequently, the patient remained in the assigned arm for follow-up at the specified time points from randomisation on the intention to treat principle.

Participants assigned to lung metastasectomy were to have surgery with the objective of an R0 resection (that is histologically confirmed clear margins). The surgical approach (videothoracoscopy or open thoracotomy) was at the discretion of the surgeon.

Patients were seen for clinical examination including performance status, weight, lung function, CEA (carcinoembryonic antigen) assay and CT at 3, 6, 9, 12, 18, 24, 36, 48 and 60 months.

\section{Outcomes}

The primary outcome was survival with all patients being followed until the date of censoring or 60 months whichever was shorter. Any surgery, radiotherapy, ablations or chemotherapy since the last report were recorded. In the event of cross-overs a secondary analysis by treatment received was to be carried out. 
Secondary endpoints were changes in lung function (FEV1 and \% predicted FEV1) and, over the period of 24 months following randomisation, patient reported anxiety and quality of life.

The primary patient reported outcome measure was the FACT An-L Trial outcome Index (TOI), which comprises the sum of scores from 37 items included in the FACT G physical ( 7 items) and functional ( 7 items) well-being subscales together with the anaemia subscale (20 items) and 3 items from the FLSI (lung) symptom index score, not represented elsewhere in the FACT G-An questionnaire. ${ }^{18-20}$

\section{Statistical Analysis}

Sample size: A 10\% difference in overall mortality at 3 years was taken to be the minimally important clinical difference for the design of the PulMiCC non-inferiority trial. Under the assumption of exponential survival curves, and with an expected three-year survival rate of $30 \%$ in the interventional arm of the trial, then a $20 \%$ survival rate for the non-interventional arm would correspond to a relative risk of death for the non- interventional vs the interventional patients of 1.3. Under the assumptions given of exponential survival and a $30 \%$ three-year survival rate in the interventional arm, a sample size of 1350 registered patients was estimated to provide 1:1 randomisation of 300 patients. This was felt to be a practical sample size although it was hoped that perhaps as many as 150 additional patients could be randomised. A sample size of 300 would provide $78 \%$ power to detect an increased relative risk of death of 1.3 for patients in the non- interventional arm, when testing at the one-sided $5 \%$ level and this was adopted as the desired sample size in the trial protocol on this basis. This corresponds to a standard error of estimation for a $10 \%$ survival difference of $4.2 \%$.

Comparative analysis: For the primary outcome of survival, Kaplan-Meier estimates of survival curves were produced. Treatment arms were compared through fitting a Cox relative risk regression model ${ }^{21}$ which provided estimated hazard ratios and confidence intervals. The primary analysis was adjusted for minimization variables. For the as-treated analysis, comparison was based on a time-dependent binary explanatory variable reflecting the time at which a metastasectomy occurred.

For lung function (FEV1 and Predicted \% FEV1) we used linear regression models with estimation using generalized estimating equations to adjust for within patient correlation. The primary analysis was to 
estimate a common effect of metastasectomy over time, with adjustment for follow-up time and baseline measurements, but variation in the treatment effect over time was examined. The potential impact of losses to follow-up was examined through fitting singular linear increment models. ${ }^{22}$ Comparable linear model methods were used for patient reported outcome data.

To reflect the high correlation between baseline and subsequent measures for all patient reported outcomes (PROs) other than the STAI (anxiety), we used the change in scores from baseline at 3, 6, 12 and 24 months. For all outcomes, models which included baseline score were fitted to examine the appropriateness of differencing. Estimated differences between the treatment arms and associated $95 \%$ confidence intervals were calculated and, where appropriate, compared with minimally important differences (MIDs). The null hypotheses addressed were of no differences in quality of life expected between metastasectomy and control. There was a particular focus on the alternatives that patients who are randomised to surgery would experience more lung function symptoms and patients who are not randomised to surgery would experience greater anxiety.

\section{Funding}

The funder of the study (Cancer Research UK) had no role in study design, data collection, data analysis, data interpretation, or writing of the report. The corresponding author had full access to all the data in the study and had final responsibility for the decision to submit.

\section{Results}

The first randomisation was Dec 2, 2010 and the last Nov 24, 2016. Recruitment slowed from 2015 and never recovered. We closed the trial in December 2016. At that time there were 512 of an intended 1350 registrations in Stage 1 and of 300 patients required by the power calculation we had randomised 93. [Fig.1] The centre in Vojvodina, Serbia failed to return CRFs because of unresolvable problems with trial support. We had only baseline data on two randomised patients, one in each group. After several discussions with the IDMC we decided to exclude the site. Remaining from 13 sites, were 65 randomised participants, 33 in the control arm and 32 assigned to metastasectomy. Apart from the excluded site in Serbia, no other patients have been lost from follow-up for the primary outcome which is survival. Ten sites registered patients but did not randomize any. The prospectively collected data on the full cohort of 512 patients and their survival will be analysed and reported separately as an observational study.

Minimisation produced balanced groups and limited the potential for unexpected confounding. [Table 1] 
Figure 2 presents estimated survival curves for the metastasectomy and control arms. The estimated hazard ratio comparing the relative survival rates in the metastasectomy versus the control treatment arm, adjusting for and therefore comparing patients with comparable minimisation variables, was 0.69 with a $95 \%$ confidence interval $(\mathrm{Cl})$ of $(0.35,1.37)$. The unadjusted estimated hazard ratio was $0.82,95 \% \mathrm{Cl}$ $(0.43,1.56)$ and unadjusted non-parametric median estimates were $3.91,95 \% \mathrm{Cl}(2.99, ¥)$, and $3.38,95 \% \mathrm{Cl}$ $(3.11, ¥)$, for the metastasectomy and control arms respectively. For the 'as treated' analyses, 2 patients assigned to metastasectomy did not have surgery. No patient in the control group crossed over to metastasectomy. The comparable adjusted and unadjusted estimated hazard ratios for these analyses were $0.60,95 \% \mathrm{Cl}(0.30,1.22)$ and $0.78,95 \% \mathrm{Cl}(0.41,1.50)$.

At five-year follow-up FEV1 related measurements were only available for 3 patients in the metastasectomy arm and 1 in the control arm. This precludes informative analysis at this time point and treatment comparisons were therefore restricted to time points up to 48 months. Based only on observed patients, for FEV1 there is no evidence of an effect with an estimated average difference over time, based on generalized estimating equations (GEE) and adjusted for baseline and follow-up time, of $-0.05,95 \% \mathrm{Cl}$ $(-0.21,0.12)$. For \% predicted FEV1, there is an estimated overall effect associated with metastasectomy of $-4.93,95 \% \mathrm{Cl}(-10.57,0.70)$. The correlations of subsequent lung function measures with baseline are 0.855 and 0.75 for FEV1 and \% predicted FEV1 respectively.

Figures 3 and 4 present estimates of the mean FEV1 and \% predicted FEV1, respectively, in the two treatment arms at various follow-up times, with a common baseline starting value assumed in both arms corresponding to the average baseline in all patients. The means are connected by straight lines for presentation purposes only. Estimates based on singular linear models that illustrate the possible effect of drop-out are presented here. It can be seen that the apparent observed increase in FEV1 and \% predicted FEV1 values at later follow-up times may be importantly influenced by drop-out. For both sets of estimates, there is an apparent cross-over of the values with the metastasectomy patients having lower values in the first year or so but showing an increase thereafter. Formal $1 \mathrm{df}$ tests, based on GEE estimation, for variation in the arm effect over (linear) time generated p-values of 0.11 and 0.02 for FEV1 and \% predicted FEV1 respectively.

There were no treatment-related deaths or major adverse events.

A total of 21 of 65 patients were reported by CRF as treated with chemotherapy over the course of the five years with no significant difference in numbers between the two arms: Control 9/33; Metastasectomy $12 / 32$. Some patients had repeated treatments. Chemotherapy within six months of randomisation was given in 5 patients in each arm. 
A total of 11 patients had radiotherapy in the five-year follow up period, 6/33 in the control group and $5 / 32$ in the metastasectomy groups, none of which were within six months of randomisation and were dispersed without a pattern thereafter. In at least one instance in each group the reported radiotherapy was to treat metastases elsewhere (brain and bone). Two patients in each group were treated with radiofrequency ablation. No treatments were in the first six months after randomisation.

All but one patient had ECOG (Eastern Cooperative Oncology Group) performance score of 0 or 1 at baseline. There is no suggestion of a difference between the arms. (Supplementary table and graphical depiction)

Figure 5 presents the mean patient reported outcome scores over the 24 months of follow-up. For no outcome was a significant effect of metastasectomy detectable. For example, for the change in TOI from baseline, the estimated effect was $-1.51,95 \% \mathrm{Cl} \quad(-.90,4.88)$. An early drop in the FLSI score (that is lung symptoms) for patients receiving surgery is observed as expected. While drop-out is significant, particularly at 24 months, linear increment analyses do not generate any qualitative difference for these outcomes. Minimal important differences for TOI, FACT-G, FACT-An-20 and FLSI, taken from the literature are 7.66, 5-7, 4.57 and 1.30 respectively. Such differences lie outside of, or towards the limit of the $95 \%$ confidence intervals for the estimated effects for these four outcomes, on the change from baseline scale, which were $(-7.90,4.88),(-5.77,3.02),(-3.94,4,45)$ and $(-1.56,0.56)$ respectively.

Variations in patients' weights in the two arms of the trial was dominated by reducing numbers and widening standard deviation. There was no discernable difference between trial arms.

\section{Discussion}

Because of recruitment difficulties PulMiCC closed early and we were unable to reach the desired statistical endpoints and convincingly answer the question about the value of pulmonary metastasectomy. Nevertheless, we believe that the results have some important implications.

The survival of patients undergoing metastasectomy in PulMiCC was similar to that found in a quantitative synthesis of all follow-up studies up to $2007^{2}$ and the meta-analysis of larger observational 
studies up to $2011 .{ }^{23}$ Five-year survival was around $40 \%$ in all three. The PulMiCC results appear to be a valid reflection of so called 'real world' practice. But survival of the PulMiCC control patients was better than is generally assumed for those with untreated colorectal lung metastases. Because of small numbers, the confidence limits are wide but the difference between the survival of those having metastasectomy and that of untreated patients with colorectal lung metastases is likely to be smaller than is currently assumed. PulMiCC is the only randomised trial of colorectal cancer metastasectomy. Because the point estimate of the HR was 0.82 our findings are compatible with the belief that some patients, in whom lung metastases are truly the only residue of their colorectal cancer, may survive long term as a direct result of metastasectomy. But they call into question the belief that there is a very low likelihood of five-year survival without metastasectomy in comparable patients.

Lung metastases generally remain asymptomatic and rarely contribute to terminal events and so there is unlikely to be significant palliative benefit from metastasectomy. There was a drop in quality of life (QoL) at three months in those assigned to operation as would be expected among patients undergoing surgery (Fig.5) and there was a detrimental effect on lung function from three months to 1-2 years after pulmonary resection compared with control. (Fig.3\&4) Neither difference was sustained or significant in the longer term, but the already small numbers of patients available for assessment declined by three years to fewer than 20 in each arm, most with ongoing cancer, making data uninterpretable. Although 'psychological benefit' is given as a justification for metastasectomy, we found no difference in anxiety between the two arms. Individual patients may have felt relieved to be rid of the radiologically visible vestiges of their cancer, but reduced anxiety was not seen as a group effect in this controlled trial. The lack of difference also indicates that informed patients can come to terms with the presence of lung metastases. This is in line with the absence of cross over from the control to the metastasectomy arm of the trial.

Comparative 'before and after' data on pulmonary function were not given in any of observational studies $^{2}$ which is consistent with the under-reporting of harms that has been found to be a feature of cancer trials. ${ }^{24}$ The overriding limitation of this study is its small size with only 65 participants. This was in large part due to the difficulty clinicians had in presenting uncertainty to patients who were referred to them in the hope of cure. ${ }^{25}$ It was also clear that the default of MDTs was to offer intervention rather than randomisation with a chance of assignment to a non-metastasectomy arm.

There are many well-documented instances where there has been a reversal from a prior standard of care after the fair test of an RCT. ${ }^{26}$ There are important precedents for finding that when subjected to a controlled trial, more radical surgery has not resulted in better cancer outcomes. ${ }^{27,28}$ Randomised trials of 
interventions, and particularly surgery versus no active treatment, are difficult to conduct and as a consequence tend to be relatively small but do provide a more reliable estimate of differences between treatments than uncontrolled observations. ${ }^{29}$ More contentious are small trials which find no difference such as the analysis of pooled trial data on 58 patients which suggested that stereotactic radiotherapy might be have similar outcomes to lobectomy in the treatment of primary lung cancer. ${ }^{30}$ PulMiCC is open to the same criticism of being small and therefore underpowered but if lung metastasectomy for colorectal cancer were not already in practice, it would not seem reasonable to propose its introduction in the light of these findings. Although not proving an absence of any survival difference, a duty of candour should include sharing with patients that there might be no benefit from metastasectomy. That alone would help in recruiting to any future trials and improve evidence for clinical practice.

Finally PulMiCC is a clear example of the difficulties of running a randomised trial that challenges established clinical practice but is based on insecure observational evidence.

\section{Declarations}

\section{Ethics approval and consent to participate}

Ethics approval was granted by the National Research Ethics Committee London - Hampstead $10 / \mathrm{H} 0720 / 5$

There was a two stage consent process for all randomised patients

\section{Consent for publication}

No patient information is disclosed

\section{Availability of data and material}

All information is freely available by application to the Chief Investigator TT and SITU UCL

\section{Competing interests}

None 


\section{Funding}

PulMICC was funded by Cancer Research UK funding Grant No. C7678/A11393

\section{Authors' contributions}

TT is chief investigator, and with VF, FM, LF and BL instigated the study

VF and OG did the statistical analysis.

BL CB-G led on trial management at Royal Brompton and UCL

NW led on data management

LF and KM planned and implemented PROMs

TT drafted and edited the manuscript with extensive input at all stages from all authors

All the authors contributed to the drafting and revision of the manuscript and have approved the manuscript.

\section{Acknowledgements}

PulMiCC was supported by the UK Thoracic Surgery Research Collaborative which includes the surgeons named as contributors. Table of Principle Investigators and numbers of patients recruited into the trial is available as supplementary material.

We are thank all the principle investigators and all members of their research teams and the patients for participating.

We are grateful to the Data Monitoring and Ethics Committee who contributed support and advice throughout the study: Professors Mike Baum (chair), Julian Peto and Martyn Evans.

\section{Abbreviations}

CEA Carcinoembryonic Antigen 


\begin{tabular}{|c|c|}
\hline CRF & Clinical Report Form \\
\hline $\mathrm{Cl}$ & Confidence Interval \\
\hline $\mathrm{CRC}$ & Colorectal Cancer \\
\hline CT & computerised tomography \\
\hline DVD & Digital Video Disc \\
\hline ECOG & Eastern Cooperative Oncology Group \\
\hline EQ-5D-3L & EuroQoL 5 Dimension 3 Level \\
\hline FACT-G-An & Functional Assessment of Cancer Therapy - General and Anaemia sub-scale \\
\hline FACT An-L & Functional Assessment of Cancer Therapy -Anaemia and Lung sub-scales \\
\hline FEV1 & Forced Expiratory Volume in the $1^{\text {st }}$ second \\
\hline FLSI & Functional Lung Symptom Index \\
\hline GEE & Generalized Estimating Equations \\
\hline IDMC & Independent Data Monitoring Committee \\
\hline IGTA & Image Guided Thermal Ablation \\
\hline MID & Minimally Important Difference \\
\hline MDT & Multidisciplinary Team \\
\hline PET & Positron Emission Tomography \\
\hline PRO & Patient Reported Outcomes \\
\hline $\mathrm{PI}$ & Principle Investigator \\
\hline PulMiCC & Pulmonary Metastasectomy in Colorectal Cancer \\
\hline QoL & Quality of Life \\
\hline RCT & Randomised Controlled Trial \\
\hline RO & No residual tumour on histological examination of the margins \\
\hline $\mathrm{E}-\mathrm{C}$ & ealth Outcomes Research \& Education in Cancer \\
\hline
\end{tabular}


SITU Surgical \& Interventional Trials Unit

STAI Spielberger State/Trait Anxiety Inventory

TMG Trial Management Group

TNM Tumour Nodal Metastasis staging system

TOI Trial Outcome Index

\section{References}

(1) Schirren J, Schirren M, Lampl L, Sponholz S. Surgery for pulmonary metastases: quo vadis? Eur J Cardiothorac Surg 2017 March 1;51(3):408-10.

(2) Fiorentino F, Hunt I, Teoh K, Treasure T, Utley M. Pulmonary metastasectomy in colorectal cancer: a systematic review and quantitative synthesis. J R Soc Med 2010 February;103(2):60-6.

(3) Pastorino U, Buyse M, Friedel G, Ginsberg RJ, Girard P, Goldstraw P et al. Long-term results of lung metastasectomy: prognostic analyses based on 5206 cases. J Thorac Cardiovasc Surg 1997;113:37-49.

(4) Aberg T, Malmberg KA, Nilsson B, Nou E. The effect of metastasectomy: fact or fiction? Ann Thorac Surg 1980 October;30(4):378-84.

(5) Barton MK. Metastasectomy on the rise across several cancer types. CA Cancer J Clin 2015 May;65(3):163-4.

(6) Bartlett EK, Simmons KD, Wachtel H, Roses RE, Fraker DL, Kelz RR et al. The rise in metastasectomy across cancer types over the past decade. Cancer 2015 March 1;121(5):747-57.

(7) Jawed I, Wilkerson J, Prasad V, Duffy AG, Fojo T. Colorectal Cancer Survival Gains and Novel Treatment Regimens: A Systematic Review and Analysis. JAMA Oncol 2015 September 1;1(6):787-95.

(8) Gonzalez M, Poncet A, Combescure C, Robert J, Ris HB, Gervaz P. Risk factors for survival after lung metastasectomy in colorectal cancer patients: a systematic review and meta-analysis. Ann Surg Oncol 2013 February;20(2):572-9.

(9) Jeffery M, Hickey BE, Hider PN, See AM. Follow-up strategies for patients treated for nonmetastatic colorectal cancer. Cochrane Database Syst Rev 2016 November 24;11:CD002200.

(10) Mokhles S, Macbeth F, Farewell V, Fiorentino F, Williams NR, Younes RN et al. Meta-analysis of colorectal cancer follow-up after potentially curative resection. Br J Surg 2016 September;103(10):125968. 
(11) Aberg T. Selection mechanisms as major determinants of survival after pulmonary metastasectomy. Ann Thorac Surg 1997 March;63(3):611-2.

(12) Glasziou P, Chalmers I, Rawlins M, McCulloch P. When are randomised trials unnecessary? Picking signal from noise. BMJ 2007 February 17;334(7589):349-51.

(13) Monson K, Treasure T, Brew-Graves C, Jenkins V, Fallowfield L.

Surveying patients' views on trial information provision and decision making using the 'Accept/Decline' clinical trials questionnaire. Trials 2017;18 (Suppl 1):24.

(14) Cella D. The Functional Assessment of Cancer Therapy-Anemia (FACT-An) Scale: a new tool for the assessment of outcomes in cancer anemia and fatigue. Semin Hematol 1997 July;34(3 Suppl 2):13-9.

(15) Cella D. FACIT questionnaire specifics manual. 24-8-2005. 24-8-2005.

Ref Type: Pamphlet

(16) Marteau TM, Bekker $\mathrm{H}$. The development of a six-item short-form of the state scale of the Spielberger State-Trait Anxiety Inventory (STAI). Br J Clin Psychol 1992 September;31 ( Pt 3):301-6.

(17) EuroQol-a new facility for the measurement of health-related quality of life. Health Policy 1990 December;16(3):199-208.

(18) Liang K, Zeger S. Longitudinal data analysis using generalized linear models. Biometrika 2018;73(1):13-22.

(19) Cella D, Nichol MB, Eton D, Nelson JB, Mulani P. Estimating clinically meaningful changes for the Functional Assessment of Cancer Therapy-Prostate: results from a clinical trial of patients with metastatic hormone-refractory prostate cancer. Value Health 2009 January;12(1):124-9.

(20) Eton D, Yost K. Minimally important differences on the functional assessment of chronic illness therapy instruments. In FACIT Manual Version 4. Section 4 - Validity and Interpretation. Doc 116. 2005. 2005.

Ref Type: Pamphlet

(21) Cox DR. Regression models and life-table. Journal of the Royal Statistical Society B 1972;34:187220.

(22) Farewell DM. Marginal analyses of longitudinal data with an informative pattern of observations. Biometrika 2010;97(1):65-78.

(23) Gonzalez M, Robert JH, Halkic N, Mentha G, Roth A, Perneger T et al. Survival after lung metastasectomy in colorectal cancer patients with previously resected liver metastases. World J Surg 
2012 February;36(2):386-91.

(24) Gyawali B, Shimokata T, Honda K, Ando Y. Reporting harms more transparently in trials of cancer drugs. BMJ 2018 November 1;363:k4383.

(25) Treasure T, Baum M. An approach to randomization into surgical clinical trials. Br J Surg 2017 January;104(1):11-2.

(26) Prasad V, Vandross A, Toomey C, Cheung M, Rho J, Quinn S et al. A decade of reversal: an analysis of 146 contradicted medical practices. Mayo Clin Proc 2013 August;88(8):790-8.

(27) Veronesi U, Saccozzi R, Del Vecchio M, Banfi A, Clemente C, De Lena M et al. Comparing radical mastectomy with quadrantectomy, axillary dissection, and radiotherapy in patients with small cancers of the breast. N Engl J Med 1981 July 2;305(1):6-11.

(28) Treasure T, Lang-Lazdunski L, Waller D, Bliss JM, Tan C, Entwisle J et al. Extra-pleural pneumonectomy versus no extra-pleural pneumonectomy for patients with malignant pleural mesothelioma: clinical outcomes of the Mesothelioma and Radical Surgery (MARS) randomised feasibility study. Lancet Oncol 2011 August;12(8):763-72.

(29) Horton R. Surgical research or comic opera: questions, but few answers. Lancet 1996 April 13;347(9007):984-5.

(30) Chang J, Senan S, Smit ERJ. Surgery versus SABR for resectable non-small cell lung cancer. Lancet Oncology 2015;16:e374-e375.

\section{Tables}

Table 1 


\section{Control $(\mathrm{N}=33) \quad$ Metastasectomy $(\mathrm{N}=32)$}

$\begin{array}{lll}\text { Male } & 20 & 21 \\ \text { Female } & 13 & 11\end{array}$

\begin{tabular}{lcc} 
Age & Years & Years \\
\hline Minimum & 48 & 35 \\
\hline $25 \%$ & 61 & 61 \\
\hline Median & 70 & 72 \\
\hline $75 \%$ & 76 & 74 \\
\hline Maximum & 86 & 83
\end{tabular}

\section{CRC T Stage}

\begin{tabular}{lcc} 
T 1 & 1 & 2 \\
\hline T2 & 4 & 4 \\
T3 & 23 & 23 \\
\hline Missing & 5 & 3 \\
Total & 33 & 32
\end{tabular}

\section{CRC N Stage}

\begin{tabular}{lcc} 
N1 & 15 & 16 \\
N2 & 13 & 13 \\
Missing & 5 & 3 \\
\hline
\end{tabular}

\section{Prior Liver resection}

$\begin{array}{lcc}\text { Yes } & 9 & 8 \\ \text { No } & 24 & 24\end{array}$

\begin{tabular}{lcc} 
CRC Interval & Months & Months \\
\hline Minimum & 7.6 & 1.0 \\
\hline $25 \%$ & 17.4 & 13.9 \\
\hline Median & 26.4 & 22.0 \\
\hline $75 \%$ & 34.8 & 36.8 \\
\hline Maximum & 130.5 & 106.5
\end{tabular}

\section{Lung metastases}

\begin{tabular}{lcc}
1 & 14 & 14 \\
2 to 4 & 18 & 16 \\
$5+$ & 1 & 2 \\
\hline
\end{tabular}

CEA ng/ml

\begin{tabular}{lcc}
$<5$ & 27 & 28 \\
\hline 5 to 10 & 2 & 1 \\
\hline $10+$ & 4 & 3
\end{tabular}

Table 1: Baseline characteristics of the intention-to-treat population.

\section{Figures}




\section{Patients consented Stage 1 PulMiCC N=512}
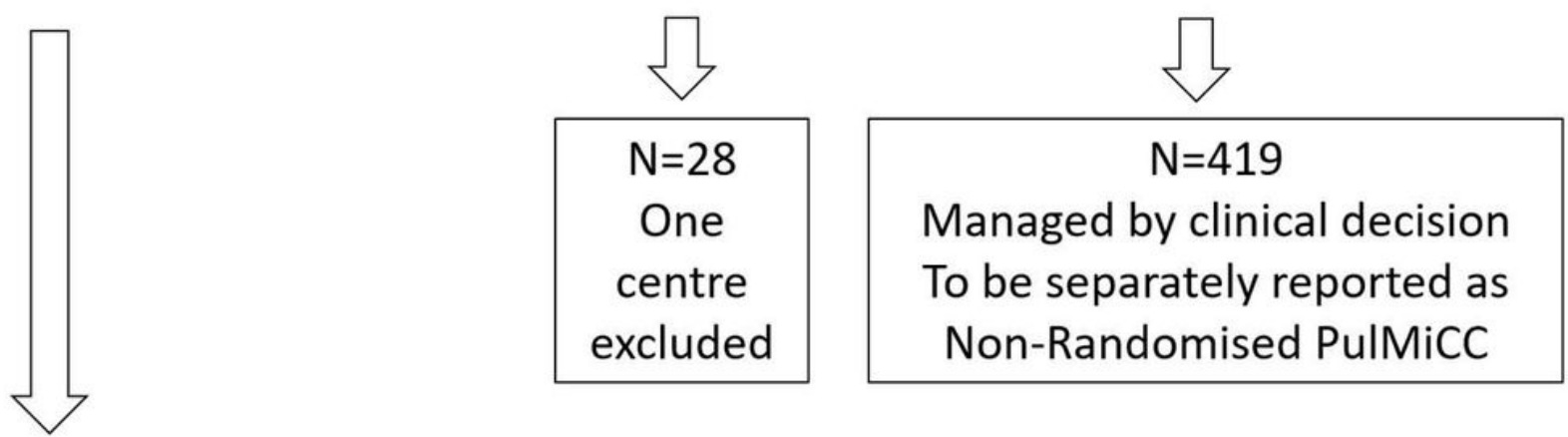

Analysable patients $\mathrm{N}=65$

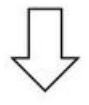

Randomised to control arm 33

33 Treated as assigned

0 Crossed over

(1 Nodules disappeared)

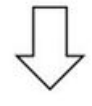

Randomised to lung metastasectomy

30 Treated as assigned

2 Crossed over

(1 Nodules were benign)

Figure 1

The PulMiCC trial profile. 


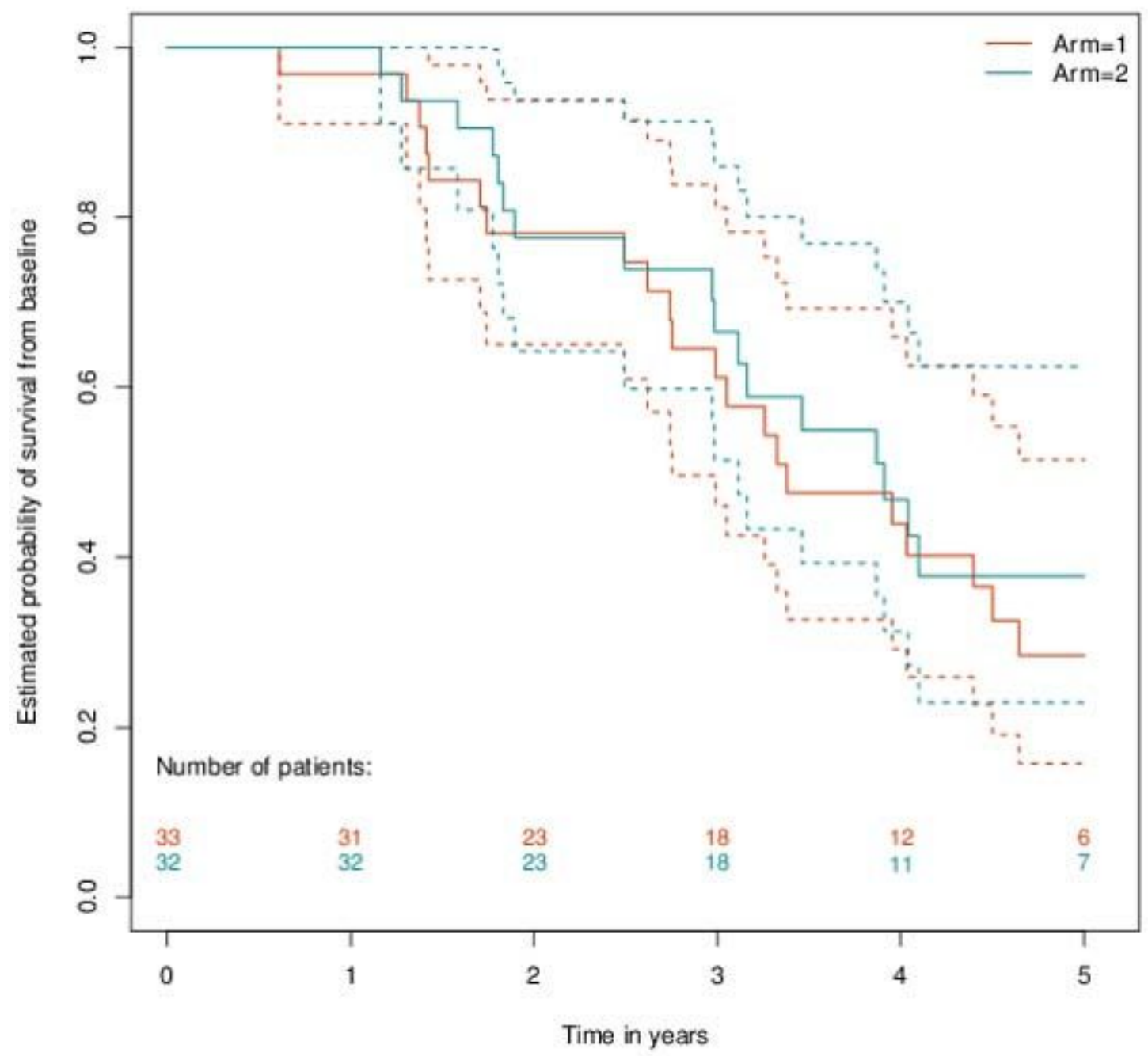

Figure 2

Kaplan Meier analysis with 95\% confidence intervals. 


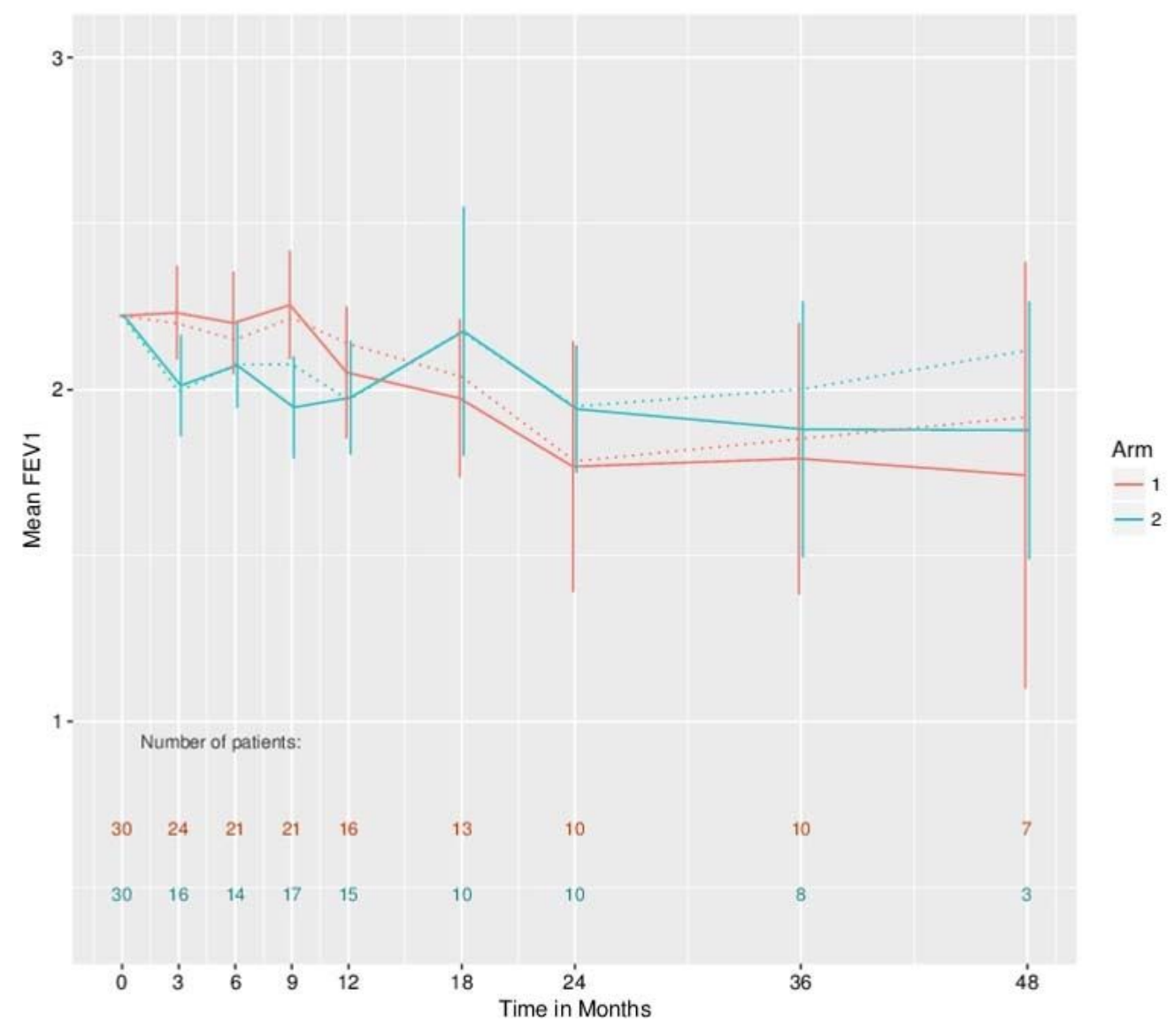

\section{Figure 3}

Estimated mean FEV1 values in the two treatment arms with a common baseline starting value assumed in both arms corresponding to the average baseline in all patients. Dashed lines based on generalized estimating equations and solid lines based on singular linear models that adjust for drop-out. 95\% Confidence intervals are provided for the singular linear model fits. 


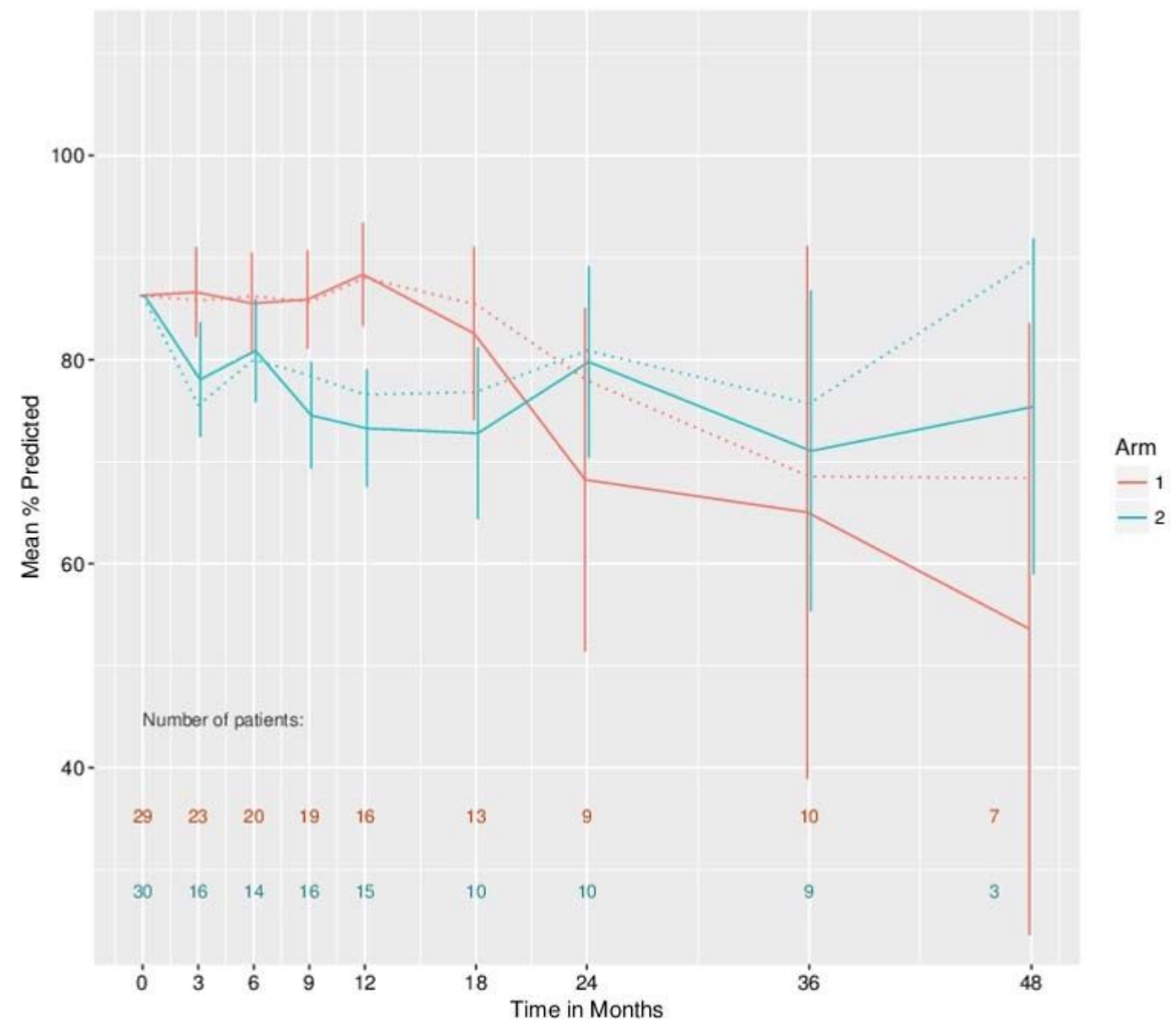

\section{Figure 4}

Estimated mean \% predicted FEV1 values in the two treatment arms with a common baseline starting value assumed in both arms corresponding to the average baseline in all patients. Dashed lines based on generalized estimating equations and solid lines based on singular linear models that adjust for drop-out. Confidence intervals are provided for the singular linear model fits. 

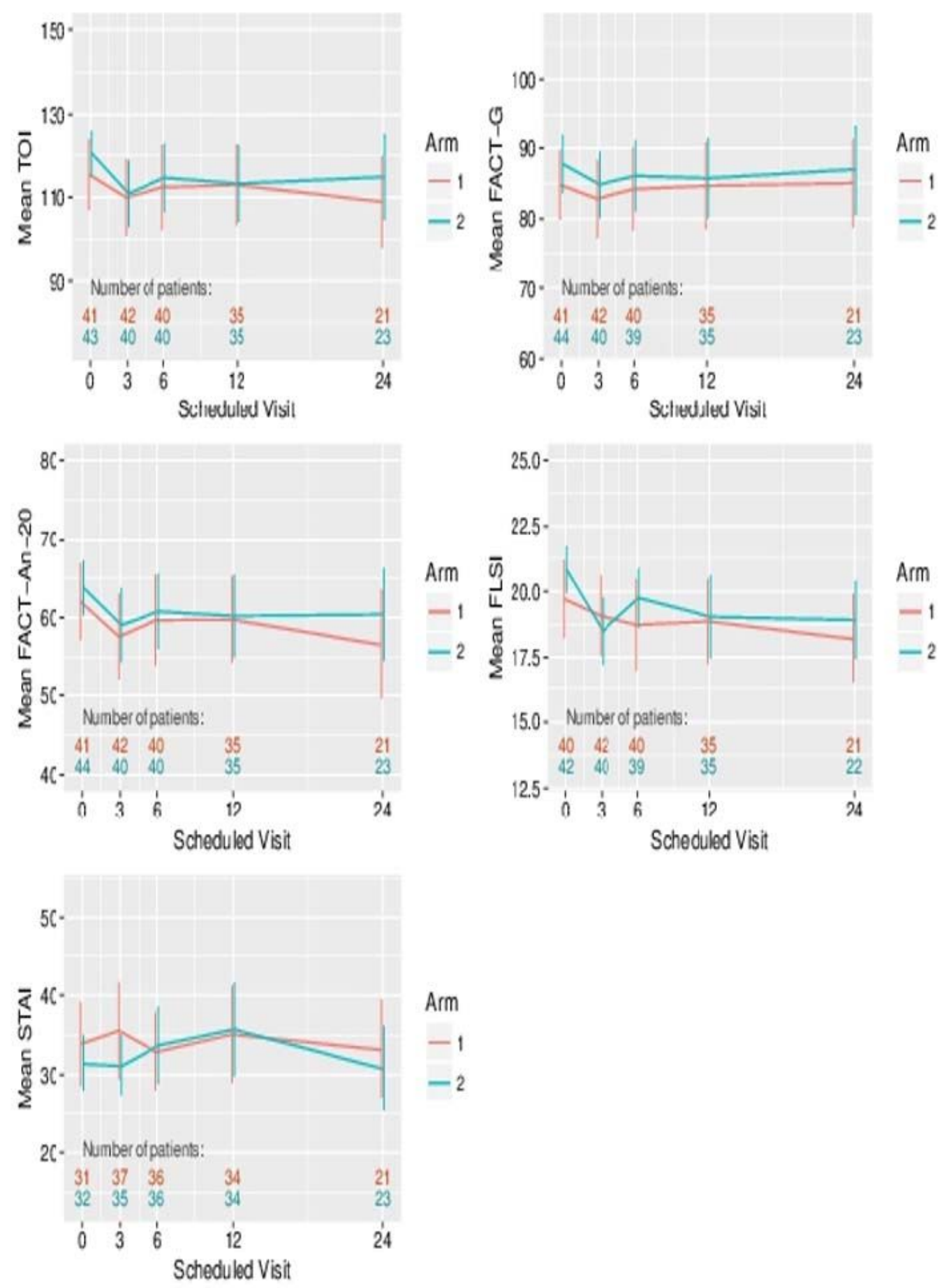

\section{Figure 5}

Patient Reported Outcomes comparing the two arms of the trial. TOI: Trial Outcome Index. Functional Assessment of Cancer Therapy (An-L) FACT-G: Functional Assessment of Cancer Therapy. General FACTAn-20: Functional Assessment of Cancer Therapy. Anaemia sub-scale) STAl: Spielberger State/Trait Anxiety Inventory FLSI: Lung Cancer Brief Symptom Index. 


\section{Supplementary Files}

This is a list of supplementary files associated with this preprint. Click to download.

- supplement1.pdf 